PROCEEDINGS OF THE

AMERICAN MATHEMATICAL SOCIETY

Volume 139, Number 5, May 2011, Pages 1825-1829

S 0002-9939(2010)10464-1

Article electronically published on July 8, 2010

\title{
PLANAR SOBOLEV HOMEOMORPHISMS AND HAUSDORFF DIMENSION DISTORTION
}

\author{
TAPIO RAJALA
}

(Communicated by Mario Bonk)

\begin{abstract}
We investigate how planar Sobolev-Orlicz homeomorphisms map sets of Hausdorff dimension less than two. With the correct gauge functions the generalized Hausdorff measures of the image sets are shown to be zero.
\end{abstract}

\section{INTRODUCTION}

We study Sobolev mappings $f \in W_{\text {loc }}^{1,1}\left(\Omega ; \mathbb{R}^{2}\right)$, where $\Omega$ is an open subset of $\mathbb{R}^{2}$. This means that the components of the mapping $f: \Omega \rightarrow \mathbb{R}^{2}$ have locally integrable distributional partial derivatives. We would like to understand how much these mappings can increase the size of small sets. There are many different ways to define the size of a set. Here we will use the standard and generalized Hausdorff measures. This approach is a natural continuation to the study of Lusin condition $(\mathrm{N})$, which requires that $\mathcal{L}^{2}(f(A))=0$ for every $A \subset \Omega$ with $\mathcal{L}^{2}(A)=0$. By $\mathcal{L}^{2}(A)$ we mean the 2-dimensional Lebesgue measure of $A$.

Without assuming $f \in W_{\text {loc }}^{1,1}\left(\Omega ; \mathbb{R}^{2}\right)$ to be a homeomorphism, Lusin condition (N) holds if we suppose that $|D f|^{p} \in L_{\text {loc }}^{1}(\Omega)$ with $p>2$; see [8]. For a homeomorphism $f \in W_{\text {loc }}^{1,1}\left(\Omega ; \mathbb{R}^{2}\right)$ a result by Reshetnyak 9 says that $f$ satisfies Lusin condition (N) under the weaker assumption $|D f|^{2} \in L_{\text {loc }}^{1}(\Omega)$. Later this was improved in [4] by showing that already $|D f|^{2} \log ^{-1}(e+|D f|) \in L_{\text {loc }}^{1}(\Omega)$ suffices for a homeomorphism $f \in W_{\text {loc }}^{1,1}\left(\Omega ; \mathbb{R}^{2}\right)$ to satisfy Lusin condition $(\mathrm{N})$.

When we replace the Lebesgue measure with Hausdorff measures we have the following result from [1: Every homeomorphism $f \in W_{\text {loc }}^{1,1}\left(\Omega ; \mathbb{R}^{2}\right)$, with $|D f|^{p} \in$ $L_{\text {loc }}^{1}(\Omega)$ for some $p>2$, maps sets of Hausdorff dimension less than two to sets of Hausdorff dimension less than two. Motivated by the results on Lusin condition $(\mathrm{N})$, here we study what happens to sets of Hausdorff dimension less than two when we assume only that $|D f|^{2} \log ^{\lambda-1}(e+|D f|) \in L_{\text {loc }}^{1}(\Omega)$ for some $\lambda>0$. It is clear that sets of Hausdorff dimension less than two can be mapped to sets of Hausdorff dimension two with such mappings; see for example 2, 5]. The usual Hausdorff measures are therefore too rough to be used to measure the image sets. This is where the generalized Hausdorff measures come into play. For them we obtain the following result.

Received by the editors October 30, 2009 and, in revised form, May 28, 2010.

2010 Mathematics Subject Classification. Primary 30C65.

The author acknowledges the support of the Academy of Finland, project No. 211229.

(C)2010 American Mathematical Society Reverts to public domain 28 years from publication 
Theorem 1.1. Let $\Omega$ be an open set in $\mathbb{R}^{2}$ and $f: \Omega \rightarrow f(\Omega)$ a homeomorphism of class $W_{\text {loc }}^{1,1}\left(\Omega ; \mathbb{R}^{2}\right)$ with

$$
|D f|^{2} \log ^{\lambda-1}(e+|D f|) \in L_{\mathrm{loc}}^{1}(\Omega)
$$

for some $\lambda>0$. Then, with $h_{\lambda}(t)=t^{2} \log ^{\lambda} \frac{1}{t}$,

$$
\mathcal{H}^{h_{\lambda}}(f(E))=0
$$

for every set $E \subset \Omega$ for which $\operatorname{dim}_{\mathrm{H}}(E)<2$.

Theorem 1.1 was proved in [5] for sets with Minkowski dimension less than two. There the result was conjectured to hold also with sets of Hausdorff dimension less than two. Theorem 1.1 proves this conjecture. The proof follows the approach used in [5]. The paper [5] also contains an example which shows that the result we prove here is sharp: There exists a homeomorphism $f$ that maps a Cantor set of Hausdorff dimension less than two to a set of positive $\mathcal{H}^{h_{\lambda}}$-measure, with $|D f|^{2} \log ^{t-1}(e+|D f|) \in L_{\text {loc }}^{1}(\Omega)$ for every $t<\lambda$.

A weaker distortion estimate was proved in $\left[6\right.$, namely that for $f \in W^{1,1}\left(\Omega ; \mathbb{R}^{2}\right)$ with $|D f|^{2} \log ^{\lambda}(e+|D f|) \in L_{\text {loc }}^{1}(\Omega)$ sets with Hausdorff dimension less than two are mapped to sets with $\mathcal{H}^{h_{\lambda}}$-measure zero.

\section{Proof of Theorem 1.1}

Let us first introduce some notation. An $r$-neighborhood of a set $A$ is written as $A+r=\{x: \operatorname{dist}(A, x)<r\}$. By $\mathcal{L}^{k}$ we mean the $k$-dimensional Lebesgue measure. We also use the generalized Hausdorff measure $\mathcal{H}^{h}$, which is defined for a set $A$ as $\mathcal{H}^{h}(A)=\lim _{\delta \rightarrow 0} \mathcal{H}_{\delta}^{h}(A)$, where

$$
\mathcal{H}_{\delta}^{h}(A)=\inf \left\{\sum_{i=1}^{\infty} h\left(\operatorname{diam} U_{i}\right): A \subset \bigcup_{i=1}^{\infty} U_{i}, \operatorname{diam}\left(U_{i}\right) \leq \delta\right\}
$$

and $h$ is a non-decreasing function with $h(0)=0$. When the gauge function is $h(t)=t^{s}$, we have the usual $s$-dimensional Hausdorff measure which we also write as $\mathcal{H}^{s}$. The Hausdorff dimension of a set $A$ is

$$
\operatorname{dim}_{\mathrm{H}}(A)=\inf \left\{s: \mathcal{H}^{s}(A)=0\right\}=\sup \left\{s: \mathcal{H}^{s}(A)=\infty\right\} .
$$

We denote the weak differential of the function $f$ by $D f: \mathbb{R}^{2} \rightarrow \mathbb{R}^{2}$ and its operator-norm as $|D f(x)|=\sup \{|D f(x) \xi|:|\xi|=1\}$. The Jacobian determinant is written as $J_{f}(x)=\operatorname{det} D f(x)$.

We are now ready to start with the proof. The main auxiliary ingredient in the proof is the following lemma from [5].

Lemma 2.1. Let $\Omega$ be an open set in $\mathbb{R}^{2}$ and $f: \Omega \rightarrow f(\Omega)$ a homeomorphism in $W_{\text {loc }}^{1,1}\left(\Omega, \mathbb{R}^{2}\right)$. Then there exists a set $F \subset f(\Omega)$ with $\mathcal{H}^{3 / 2}(F)=0$ such that for all $y \in f(\Omega) \backslash F$ there exist constants $C_{y}>0$ and $r_{y}>0$ such that

$$
\operatorname{diam}\left(f^{-1}(B(y, r))\right) \leq C_{y} r^{1 / 2}
$$

for all $0<r<r_{y}$.

Using the previous lemma we are able to cover almost all of the target $f(\Omega)$ with a countable collection of sets where the homeomorphism $f$ maps uniformly nicely. 
Lemma 2.2. Let $\Omega$ be an open set in $\mathbb{R}^{2}$ and $f: \Omega \rightarrow f(\Omega)$ a homeomorphism in $W_{\text {loc }}^{1,1}\left(\Omega, \mathbb{R}^{2}\right)$. Then there exists a decomposition $f(\Omega)=\bigcup_{i=0}^{\infty} F_{i}$, where $\mathcal{H}^{3 / 2}\left(F_{0}\right)=$ 0 and where for each $F_{i}, i=1,2, \ldots$, there exist constants $1 \leq C_{i}<\infty$ and $R_{i}>0$ such that

$$
f^{-1}\left(\left(f(A) \cap F_{i}\right)+\left(\frac{r}{C_{i}}\right)^{2}\right) \subset A+r
$$

for every $A \subset \Omega$ and every $r \in\left(0, R_{i}\right)$.

Proof. As in [5, Lemma 3.2] we may represent $f(\Omega)$ as

$$
f(\Omega)=F_{0} \cup \bigcup_{j=1}^{\infty} \bigcup_{k=1}^{\infty}\left\{y \in f(\Omega) \mid \operatorname{diam}\left(f^{-1}(B(y, r))\right) \leq k r^{\frac{1}{2}} \text { for all } r \in\left(0, \frac{1}{j}\right)\right\},
$$

where $F_{0}=F$ is the set in Lemma 2.1.

Proof of Theorem 1.1. Take $s$ so that $\operatorname{dim}_{\mathrm{H}}(E)<s<2$. Let $F_{i}, C_{i}$ and $R_{i}$ be as in Lemma 2.2. We will show that $\mathcal{H}^{h_{\lambda}}\left(F_{i} \cap f(E)\right)=0$ for all $i \in \mathbb{N}$. This clearly holds for $F_{0}$. Let $i \geq 1$.

Because we are considering a homeomorphism $f \in W_{\text {loc }}^{1,1}\left(\Omega, \mathbb{R}^{2}\right)$, its Jacobian is either non-negative almost everywhere in $\Omega$ or non-positive almost everywhere in $\Omega$; see for example [7, Chapter 1]. We may assume that $J_{f} \geq 0$ almost everywhere in $\Omega$. Because

$$
|D f|^{2} \log ^{\lambda-1}(e+|D f|) \in L_{\mathrm{loc}}^{1}(\Omega)
$$

we have by [3, Corollary 9.1] that $J_{f} \log ^{\lambda}\left(e+J_{f}\right) \in L_{\text {loc }}^{1}(\Omega)$. Now take $\epsilon>0$. By the absolute continuity of the integral there is $\delta>0$ so that

$$
\int_{A} J_{f}(x) \log ^{\lambda}\left(e+J_{f}(x)\right) d x<\epsilon
$$

for every $A \subset \Omega$ for which $\mathcal{L}^{2}(A)<\delta$. Write $\sigma=\frac{2-s}{2}$. Let $0<r_{0}<1$ be so small that $2 \log ^{\lambda}\left(\frac{C_{i}}{r}\right) \leq r^{-\sigma}$ for all $0<r<r_{0}$. Because $\mathcal{H}^{s}(E)=0$, there exists a countable collection of balls $\left\{B\left(x_{j}, r_{j}\right)\right\}_{j=1}^{\infty}$ with radii less than $\min \left\{r_{0}, R_{i}, \frac{1}{C_{i}}\right\}$ so that

$$
\sum_{j=1}^{\infty} 4 \pi r_{j}^{s}<\min \{\epsilon, \delta\}
$$

and $E \subset \bigcup_{j=1}^{\infty} B\left(x_{j}, r_{j}\right)$.

Now write $F_{i, j}=F_{i} \cap f\left(B\left(x_{j}, r_{j}\right)\right)$ for every $j \in \mathbb{N}$. Then by Lemma 2.2 we have $f^{-1}\left(F_{i, j}+R_{i, j}\right) \subset B\left(x_{j}, 2 r_{j}\right)$, where $R_{i, j}=\left(\frac{r_{j}}{C_{i}}\right)^{2} \leq r_{0}$.

Next we use the $5 r$-covering theorem on the target side to find a pairwise disjoint collection of balls $\left\{B\left(y_{k}, R_{i, j(k)}\right)\right\}_{k \in K}$ from

$$
\left\{B\left(x, R_{i, j}\right): x \in F_{i, j}, j \in \mathbb{N}\right\}
$$

so that

$$
F_{i} \cap f(E) \subset \bigcup_{k \in K} B\left(y_{k}, 5 R_{i, j(k)}\right) .
$$


By estimating that any ball of radius $10 r$ in $\mathbb{R}^{2}$ can be covered by 1000 balls of radius $r$ we see that

$$
\begin{aligned}
& \frac{1}{1000} \mathcal{H}_{r_{0}}^{h_{\lambda}}\left(F_{i} \cap f(E)\right) \leq \sum_{k \in K} R_{i, j(k)}^{2} \log ^{\lambda}\left(\frac{1}{R_{i, j(k)}}\right) \\
& \leq \sum_{k \in K} \mathcal{L}^{2}\left(B\left(y_{k}, R_{i, j(k)}\right)\right) \log ^{\lambda}\left(\frac{1}{R_{i, j(k)}}\right) \\
& \leq \sum_{k \in K} \int_{f^{-1}\left(B\left(y_{k}, R_{i, j(k)}\right)\right)} \log ^{\lambda}\left(\frac{1}{R_{i, j(k)}}\right) J_{f}(x) d x \\
& \leq \sum_{k \in K}\left(\int_{\left\{z \in f^{-1}\left(B\left(y_{k}, R_{i, j(k)}\right)\right): J_{f}(z)<r_{j(k)}^{-\sigma}\right\}} \log ^{\lambda}\left(\frac{1}{R_{i, j(k)}}\right) J_{f}(x) d x\right. \\
&\left.+\int_{\left\{z \in f^{-1}\left(B\left(y_{k}, R_{i, j(k)}\right)\right): J_{f}(z) \geq r_{j(k)}^{-\sigma}\right\}} \log ^{\lambda}\left(\frac{1}{R_{i, j(k)}}\right) J_{f}(x) d x\right) \\
& \leq \sum_{k \in K} r_{j(k)}^{-2 \sigma} \mathcal{L}^{2}\left(f^{-1}\left(B\left(y_{k}, R_{i, j(k)}\right)\right)\right) \\
&+\sum_{k \in K} \frac{\log ^{\lambda}\left(1 / R_{i, j(k)}\right)}{\log ^{\lambda}\left(e+1 / r_{j(k)}^{\sigma}\right)} \int_{f^{-1}\left(B\left(y_{k}, R_{i, j(k)}\right)\right)} J_{f}(x) \log ^{\lambda}\left(e+J_{f}(x)\right) d x
\end{aligned}
$$

where in the third inequality we have used [4, Lemma 3.2] and in the last one the fact that $\log ^{\lambda}\left(\frac{1}{R_{i, j(k)}}\right)=2 \log ^{\lambda}\left(\frac{C_{i}}{r_{j(k)}}\right) \leq r_{j(k)}^{-\sigma}$.

Our task is to estimate the two remaining sums. By grouping the balls according to $j(k)$, we get for the first sum an estimate

$$
\begin{aligned}
\sum_{k \in K} r_{j(k)}^{-2 \sigma} \mathcal{L}^{2}\left(f^{-1}\left(B\left(y_{k}, R_{i, j(k)}\right)\right)\right) & =\sum_{j=1}^{\infty} r_{j}^{s-2} \sum_{\substack{k \in K \\
j(k)=j}} \mathcal{L}^{2}\left(f^{-1}\left(B\left(y_{k}, R_{i, j}\right)\right)\right) \\
& \leq \sum_{j=1}^{\infty} r_{j}^{s-2} \mathcal{L}^{2}\left(B\left(x_{j}, 2 r_{j}\right)\right)=\sum_{j=1}^{\infty} 4 \pi r_{j}^{s}<\epsilon .
\end{aligned}
$$

To estimate the second sum, first note that for every $k \in K$ we have

$$
\frac{\log ^{\lambda}\left(1 / R_{i, j(k)}\right)}{\log ^{\lambda}\left(e+1 / r_{j(k)}^{\sigma}\right)}=\frac{2^{\lambda} \log ^{\lambda}\left(C_{i} / r_{j(k)}\right)}{\log ^{\lambda}\left(e+1 / r_{j(k)}^{\sigma}\right)} \leq \frac{2^{\lambda} \log ^{\lambda}\left(1 / r_{j(k)}^{2}\right)}{\log ^{\lambda}\left(1 / r_{j(k)}^{\sigma}\right)}=\frac{4^{\lambda}}{\sigma^{\lambda}} .
$$

From this we get

$$
\begin{aligned}
\sum_{k \in K} \frac{\log ^{\lambda}\left(1 / R_{i, j(k)}\right)}{\log ^{\lambda}\left(e+1 / r_{j(k)}^{\sigma}\right)} & \int_{f^{-1}\left(B\left(y_{k}, R_{i, j(k)}\right)\right)} J_{f}(x) \log ^{\lambda}\left(e+J_{f}(x)\right) d x \\
& \leq \frac{4^{\lambda}}{\sigma^{\lambda}} \sum_{k \in K} \int_{f^{-1}\left(B\left(y_{k}, R_{i, j(k)}\right)\right)} J_{f}(x) \log ^{\lambda}\left(e+J_{f}(x)\right) d x \\
& \leq \frac{4^{\lambda}}{\sigma^{\lambda}} \int_{\bigcup_{k \in K} f^{-1}\left(B\left(y_{k}, R_{i, j(k)}\right)\right)} J_{f}(x) \log ^{\lambda}\left(e+J_{f}(x)\right) d x \\
& \leq \frac{4^{\lambda}}{\sigma^{\lambda}} \int_{\bigcup_{j=1}^{\infty} B\left(x_{j}, 2 r_{j}\right)} J_{f}(x) \log ^{\lambda}\left(e+J_{f}(x)\right) d x \leq \frac{4^{\lambda}}{\sigma^{\lambda}} \epsilon,
\end{aligned}
$$


since the sets $\left\{f^{-1}\left(B\left(y_{k}, R_{i, j(k)}\right)\right)\right\}_{k \in K}$ are pairwise disjoint and

$$
\mathcal{L}^{2}\left(\bigcup_{j=1}^{\infty} B\left(x_{j}, 2 r_{j}\right)\right) \leq \sum_{j=1}^{\infty} 4 \pi r_{j}^{2} \leq \sum_{j=1}^{\infty} 4 \pi r_{j}^{s}<\delta .
$$

The proof is now finished, as we have shown that

$$
\mathcal{H}_{r_{0}}^{h_{\lambda}}\left(F_{i} \cap f(E)\right) \leq 1000\left(1+\frac{4^{\lambda}}{\sigma^{\lambda}}\right) \epsilon
$$

for every $r_{0}, \epsilon>0$ and $i \in \mathbb{N}$.

\section{ACKNOWLEDGMENT}

The author thanks Aleksandra Zapadinskaya for pointing out a mistake in an earlier version of the manuscript.

\section{REFERENCES}

1. F. W. Gehring and J. Väisälä, Hausdorff dimension and quasiconformal mappings, J. London Math. Soc. (2) 6 (1973), 504-512. MR0324028 (48:2380)

2. D. A. Herron and P. Koskela, Mappings of finite distortion: gauge dimension of generalized quasicircles, Illinois J. Math. 47 (2003), no. 4, 1243-1259. MR2037001 (2005a:30031)

3. T. Iwaniec and A. Verde, A study of Jacobians in Hardy-Orlicz spaces, Proc. Roy. Soc. Edinburgh Sect. A 123 (1999), no. 3, 539-570. MR1693625 (2000g:42025)

4. J. Kauhanen, P. Koskela and J. Malý, Mappings of finite distortion: condition N, Michigan Math. J. 49 (2001), no. 1, 169-181. MR1827080 (2002d:30027)

5. P. Koskela, A. Zapadinskaya and T. Zürcher, Generalized dimension distortion under planar Sobolev homeomorphisms, Proc. Amer. Math. Soc. 137 (2009), no. 11, 3815-3821. MR2529891

6. P. Koskela, A. Zapadinskaya and T. Zürcher, Mappings of finite distortion: generalized Hausdorff dimension distortion, J. Geom. Anal. 20 (2010), no. 3, 690-704.

7. O. Lehto and K. I. Virtanen, Quasiconformal mappings in the plane, Springer-Verlag, New York, 1973. MR0344463 (49:9202)

8. M. Marcus and V. Mizel, Transformations by functions in Sobolev spaces and lower semicontinuity for parametric variational problems, Bull. Amer. Math. Soc. 79 (1973), 790-795. MR0322651 (48:1013)

9. Yu. G. Reshetnyak, Some geometrical properties of functions and mappings with generalized derivatives, Sibirsk. Math. Zh. 7 (1966), 886-919. MR.0203013 (34:2872)

Department of Mathematics and Statistics, P.O. Box 35 (MaD), FI-40014 University OF JYVÄSKYLÄ, FinLAND

E-mail address: tapio.m.rajala@jyu.fi 